\title{
COMITÉS DE EVALUACIÓN ÉTICA Y CIENTÍFICA PARA LA INVESTIGACIÓN EN SERES HUMANOS Y LAS PAUTAS CIOMS 2002
}

\author{
Eduardo Rodríguez Yunta*
}

\begin{abstract}
Resumen: El presente artículo pretende ser una reflexión y guía para el trabajo de evaluación de protocolos de investigación en seres humanos que deben realizar los comités de evaluación ética y científica teniendo en cuenta el contexto de Latinoamérica, según se desprende de las normas CIOMS 2002, recientemente traducidas por la Unidad de Bioética de la Organización Panamericana de la Salud. Se proporcionan recomendaciones para la evaluación siguiendo los requisitos éticos de la investigación en seres humanos propuestos por Ezequiel Emanuel: valor, validez científica, selección equitativa del sujeto, proporción favorable de riesgo-beneficio, evaluación independiente, consentimiento informado y respeto por los sujetos inscritos.
\end{abstract}

Palabras Clave: Ética, investigación, comités

\section{COMMITTEES OF ETHICAL AND SCIENTIFIC EVALUATION FOR RESEARCH IN HUMAN BEINGS AND THE GUIDELINES CIOMS 2002}

\begin{abstract}
The present paper tries to be a reflection and guide for the evaluation of research proposals involving human beings by ethical review committees, considering the Latin American context, as presented by CIOMS Guidelines 2002, recently translated into Spanish by the Bioethics Unit of the Pan American Health Organization. Recommendations for review are presented according to the ethical requirements for research involving human beings proposed by Ezequiel Emanuel: Value, scientific validity, fair subject selection, favorable risk-benefit selection, independent review, informed consent, respect for enrolled subjects.
\end{abstract}

Key Words: Ethics, research, committees

\section{COMITÊS DE AVALIAÇÃO ÉTICA E CIENTÍFICA PARA A PESQUISA EM SERES HUMANOS E AS DIRETRIZES CIOMES 2002}

Resumo: O presente artigo quer ser uma reflexão e guia para o trabalho de avaliação de protocolos de pesquisa em seres humanos que devem realizar os comitês de avaliação ética e científica, levando em conta o contexto da América Latina, conforme de deduz das normas CIOMS 2002, recentemente traduzidas pela Unidade de Bioética da Organização Panamericana da Saúde. São apresentadas recomendações para a avaliação, segundo as exigências éticas da pesquisa em seres humanos propostos por Ezequiel Emanuel: valor, validez científica, seleção eqüitativa do sujeito, proporção favorável de risco-benefício, avaliação independente, consentimento informado e respeito pelos sujeitos inscritos.

Palavras chave: Ética, pesquisa, comitês

\footnotetext{
* Doctor en Biología Celular y Molecular, Master en Teología, Especialidad Ética. Investigador del Centro Interdisciplinario de Estudios en Bioética, Universidad de Chile

Correspondencia: rodrigue@chi.ops-oms.org
} 


\section{Introducción}

El Consejo de Organizaciones Internacionales de Ciencias Médicas (CIOMS) ha establecido pautas desde 1982 para guiar la implementación de principios éticos que rijan la conducta de la investigación en seres humanos, según lo indica la Declaración de Helsinki, al ser aplicados en los países en desarrollo, teniendo en cuenta sus condiciones socioeconómicas y las leyes, regulaciones y disposiciones ejecutivas y administrativas. Con el establecimiento de normas de acción trata de que se respeten los principios éticos, internacionalmente, de tal manera que no haya abuso por parte de los países desarrollados. Su última versión apareció en $2002^{1}$. Estas pautas se refieren no solamente a investigación biomédica con seres humanos, sino también a la sociológica. Se considera como investigación todo estudio cuyo propósito sea producir nuevo conocimiento generalizable, incluyendo las investigaciones de estudios médicos (procesos; respuesta a intervenciones; ensayos controlados de intervenciones diagnósticas, preventivas o terapéuticas; determinación de consecuencias de intervenciones preventivas o terapéuticas) y de comportamiento relativos a la salud humana ${ }^{2}$. Se diseña el protocolo de investigación para aumentar el conocimiento objetivo de la realidad por medio de teorías, principios y relaciones de causalidad, que pueden ser corroborados y aceptados por la observación y la inferencia científica.

Los principios éticos que rijan la operación de los comités de evaluación ética y científica deben ser los mismos, independientemente de

Consejo de Organizaciones Internacionales de Ciencias Médicas. Pautas Éticas Internacionales para la Investigación Biomédica en Seres Humanos. Santiago de Chile: Programa Regional de Bioética OPS/OMS; 2003.

2 Council for International Organizations of Medical Sciences. International Ethical Guidelines for Biomedical Research Involving Human Subjects. Geneva: CIOMS; 2002: Preamble. que sean institucionales o pertenezcan a un órgano del gobierno e independientemente del país donde se realice, con la salvedad de que los comités han de operar dentro de políticas nacionales y locales. Es un requisito de cualquier investigación que involucre seres humanos que deba ser sometida a una evaluación ética y científica, por lo que es imprescindible la formación de comités de evaluación. Estos comités han de regirse por normas internas, servir para el desarrollo educacional ético de la institución a la que pertenecen, han de tener una constitución equilibrada en cuanto a miembros y han de ser capaces de evaluar ética y científicamente los proyectos que se presenten, para lo cual todavía falta mucho camino en la situación latinoamericana en general. Se puede definir un comité de ética en investigación en seres humanos como un grupo pluridisciplinar de personas idóneas y responsables, que se ocupa de abordar metódica y sistemáticamente las cuestiones éticas que surgen con la investigación en seres humanos de naturaleza biomédica, psicológica o social; formular directivas y tomar las decisiones que autoricen la realización de una determinada investigación o, en su defecto, que sea modificado el protocolo propuesto por los investigadores para que se ajuste a los principios éticos ${ }^{3}$. Estos comités tienen poder de veto, en el sentido de que pueden rechazar protocolos de investigación por no cumplir con las pautas éticas establecidas. En general, se necesita que la investigación sea evaluada en dos niveles que, aunque relacionados, son diferentes: la evaluación ética y la evaluación de la validez y significación científicas, lo cual puede realizarse por un comité capacitado para las dos evaluaciones o por separado: un subcomité de evaluación ética y otro que evalúe la parte estrictamente científica. En cuanto a su relación, la evaluación ética está ligada direc-

Reich W. Perché I comitati di etica? Una valutazione dei diversi modelli. En: Spinsanti S. I Comitati di Etica in Ospedale. Roma: Ed. Paoline; 1988: 19. 
tamente a la científica. Una propuesta de investigación en seres humanos que no sea válida científicamente no puede ser ética ${ }^{4}$. En el caso de investigación patrocinada externamente, las pautas determinan que debe haber evaluación ética y científica, tanto en el país patrocinador como en el anfitrión; los estándares éticos aplicados no deben ser menos exigentes que los establecidos para la investigación realizada en el país patrocinador, y el país anfitrión tiene la responsabilidad de determinar que los objetivos de la investigación respondan a las necesidades y prioridades de salud del país y de la población y comunidad donde se desarrollarás . También, cualquier intervención o producto desarrollado, o conocimiento generado, se ha de poner razonablemente a disposición para beneficio de la población o comunidad ${ }^{6}$.

El presente trabajo pretende ser una reflexión y guía en relación con los comités de evaluación ética y científica de acuerdo con las pautas CIOMS 2002 y los principios éticos.

\section{Composición de los Comités}

Los comités pueden ser de carácter nacional o local. Entre sus características imprescindibles están ${ }^{7}$ :

1. La independencia. Los comités de evaluación deben ser independientes de los investigadores que lo solicitan para evitar conflictos de intereses.

2. La multidisciplinariedad. Entre los miembros de un comité de evaluación científica debe incluirse el suficiente número de expertos que cubra todas las áreas que apare-

\footnotetext{
Council for International Organizations of Medical Sciences. International Ethical Guidelines for Biomedical Research Involving Human Subjects. Geneva: CIOMS; 2002: Guidelines 1 and 2.

Ibíd, Guidelines 3 and 10.

Ibíd, Guideline 10.

Ibíd, Guideline 2.
}

cen en el diseño de la investigación propuesta, por ejemplo, profesionales de las áreas de estadística, epidemiología, biología molecular, ciencias sociales y del campo de la salud. Siguiendo las pautas, entre los miembros de un comité de evaluación ética debe incluirse a científicos, médicos, abogados, profesionales de la salud, especialistas en ética, religiosos y miembros legos calificados para representar los valores culturales y morales de la comunidad, y asegurar que los derechos de los sujetos serán respetados. Deben estar representados, tanto hombres como mujeres, y un rango suficiente de personas que interpreten los diferentes valores culturales y éticos en una sociedad pluralista. La presencia de estas personas legas ayuda a entender aspectos comunitarios de costumbres y tradiciones que podrían entrar en conflicto con el tipo de investigación que se propone, de forma que siempre se han de respetar los derechos y el bienestar de las personas que van a ser potenciales sujetos; asimismo, puede variar la forma de obtener consentimiento informado, lo que puede ser considerado como incentivo indebido, y la forma de salvaguardar la confidencialidad de datos que pueden ser considerados más o menos privados o sensitivos para las diferentes culturas. Los comités también deben asegurar que haya una forma establecida de control de los datos durante el desarrollo de la investigación.

Otra regla en la formación de los comités, sea de evaluación científica o ética, establece que los miembros que los constituyen deben cambiar periódicamente ${ }^{8}$. Esto permite dar oportunidad a un mayor número de personas para que participen de esta actividad y evita que se forme un grupo de elite que termine dictando las normas. Para conjugar la experiencia con

Ibíd, Guideline 2 . 
la renovación se puede instaurar un sistema de rotación, de forma que siempre quede un grupo que ya tenga experiencia y los miembros participen del suficiente tiempo como para adquirir experiencia en la evaluación.

Los comités deben establecer reglas como elegibilidad de los miembros, número mínimo para obtener quorum, frecuencia de las reuniones, procedimientos de toma de decisiones y evaluación de decisiones.

Los comités no solamente tienen la función de evaluar las propuestas de investigación presentadas, sino, también, de realizar actividades educativas relacionadas con la ética.

\section{Requisitos Éticos de la Investigación en Seres Humanos}

Para ayudar a evaluar la ética de las propuestas de investigación que involucran a seres humanos, seguimos los siete requisitos éticos propuestos por Ezekiel Emanuel ${ }^{9}$ e incluimos, en cada uno, los aspectos que son considerados por las pautas CIOMS 2002:

\section{Valor}

Se trata de emitir un juicio sobre la importancia social, científica o clínica de la investigación ${ }^{10}$. Se evalúa que la intervención conduzca a mejoras en la salud, bienestar, genere conocimiento, sea un estudio preliminar para desarrollar una intervención o probar una hipótesis. Las razones para emitir este juicio confirman que los recursos son limitados (dinero, espacio, tiempo) y, por tanto, es necesario que

Emanuel E. ¿Qué hace que la investigación clínica sea ética? Siete requisitos éticos. En: Pellegrini A, Macklin R, eds. Investigación en Sujetos Humanos: Experiencia Internacional. Santiago de Chile: Programa Regional de Bioética OPS/ OMS; 1999: 33-46.

10 Council for International Organizations of Medical Sciences. International Ethical Guidelines for Biomedical Research Involving Human Subjects. Geneva: CIOMS; 2002: Guideline 2. exista un uso responsable de la investigación sin que suponga un gasto indebido para la sociedad; se debe evitar la explotación innecesaria de personas sujetos de investigación y no exponer a los seres humanos a riesgos y daños potenciales a menos que se esperen resultados valiosos de beneficio personal o social.

Se debe justificar la necesidad de que se lleve a cabo en seres humanos, habiendo pasado pruebas de laboratorio $\mathrm{y} / \mathrm{o}$ en animales ${ }^{11}$.

\section{Validez cientifica}

Para que haya validez científica se debe vigilar que:

A. El estudio sea original y significativo científicamente; exista idoneidad del protocolo en relación con los objetivos del estudio; sea prácticamente realizable; pueda alcanzar conclusiones válidas con la menor exposición posible de los sujetos; posea diseño, objetivo y metodología científica válidos, y un plan de análisis de datos ${ }^{12}$.

B. Se garantice que los profesionales que realicen el estudio tengan suficiente competencia científica en cuanto a experiencia y entrenamiento en las técnicas que se usarán ${ }^{13}$. Se han de tener en cuenta las obligaciones de los investigadores y el trabajo realizado previamente.

C. Se valide que las condiciones del lugar donde se lleva a cabo la investigación sean adecuadas $^{14}$.

D. Haya provisión de toma de registros y medidas para evaluar el progreso y la calidad de la investigación ${ }^{15}$.

\footnotetext{
Ibíd, Guideline 2.

Ibíd, Guidelines 1 and 2.

Ibíd, Guideline 1 .

Ibíd, Guideline 2 .

Ibíd, Guideline 2.
} 
E. Se justifique científicamente la necesidad y aceptabilidad del uso de controles alternativos a intervenciones de efectividad comprobada, en el caso de investigaciones clínicas que los empleen ${ }^{16}$.

F. Se asegure que la publicación de los resultados de la investigación sea veraz, consistente y justa ${ }^{17}$.

\section{Selección equitativa del sujeto}

La identificación y selección de potenciales sujetos debe ser equitativa en cuanto a la distribución de cargas y beneficios ${ }^{18}$. Esto se justifica por el principio de la equidad distributiva: los beneficios y cargas de la vida social deben ser distribuidos equitativamente. Para ello se debe cuidar que:

A. Se seleccionen grupos específicos relacionados con la interrogante científica de la investigación. Evitar, si no es imprescindible, la elección de grupos vulnerables (estigma, impotencia), a menos que sea necesario para la naturaleza de la investigación. Las pautas consideran vulnerable al sujeto que tiene una incapacidad sustancial para proteger intereses propios debido a impedimentos como: ineptitud para dar consentimiento informado, falta de medios alternativos para conseguir atención médica u otras necesidades de alto costo, o ser un miembro subordinado de un grupo jerárquico ${ }^{19}$. Son grupos vulnerables en la investigación los discapacitados, niños, ancianos, enfermos mentales, presos, minorías y poblaciones de países subdesarrollados en quienes se realizan proyectos que no se pueden llevar a cabo en los países industrializados.
B. A todos los grupos se les ofrezca oportunidad de participar, a menos que presenten factores de riesgo que restrinjan su elegibili$\mathrm{dad}^{20}$.

C. Los seleccionados estén en condiciones de beneficiarse si la investigación proporciona un resultado positivo (aplicación a la salud y bienestar de grupos específicos). A menos que hubiera razones científicas o potencial de daño para excluirlos ${ }^{21}$.

D. La selección de sujetos esté diseñada para reducir al mínimo los riesgos, a la vez que se maximicen los beneficios sociales y científicos de los resultados de la investigación $^{22}$.

E. Se justifique la exclusión de grupos o comunidades y la inclusión de grupos vulnerables $^{23}$. El uso de sujetos vulnerables queda debidamente fundado si se demuestra que el proyecto de investigación beneficia directamente a la población de posibles sujetos, hubo consulta comunitaria y fue aceptado, y se incluye a los menos vulnerables del grupo o población ${ }^{24}$. En el caso de enfermos mentales, se obtiene consentimiento en períodos lúcidos de los pacientes ${ }^{25}$.

F) Se seleccione el número mínimo de sujetos suficiente para realizar análisis estadístico ${ }^{26}$.

G) Se acepte la investigación biomédica de mujeres en edad reproductiva ${ }^{27}$ y de mujeres embarazadas ${ }^{28}$, siempre que se informe de los posibles peligros asociados, haya consentimiento informado, atención médica re-
Ibíd, Guideline 8 .
Ibíd, Guidelines 8 and 10.
Ibíd, Guideline 8.
Ibíd, Guideline 13.
Ibíd, Guideline 13.
Ibíd, Guideline 15.
Ibíd, Guideline 2.
Ibíd, Guideline 16.
Ibíd, Guideline 17. 
querida, se respeten los valores de los potenciales sujetos, no se exponga a riesgos innecesarios a la mujer o al feto.

\section{Proporción favorable de riesgo-beneficio}

Desde el punto de vista ético, siempre es posible que los pacientes decidan perjudicando a sus propios intereses y bienestar. Por este motivo, por los principios de beneficencia y no maleficencia, se debe establecer límites a la posibilidad de que los potenciales sujetos de investigación acepten riesgos desproporcionados. En ese sentido, al comité de ética en investigación y al equipo de investigadores les corresponde evaluar cada protocolo ponderando si en él:

\section{A. Los riesgos potenciales a los sujetos indivi-} duales se minimizan ${ }^{29}$. Es preciso justificar los riesgos que no son evitables, puesto que se respeta la integridad física y emocional de los sujetos de investigación; en consecuencia, se debe cuidar que el riesgo no sea superior al mínimo. Se estima que un riesgo es mínimo cuando el estudio es de tales características, que la probabilidad y magnitud del daño o malestar previsto en la investigación no es mayor, en sí mismo, que aquellos comúnmente afrontados en la vida cotidiana o durante el desempeño de exámenes o pruebas físicas o psicológicas de rutina. El investigador tiene que ponderar los riesgos potenciales y beneficios para el sujeto; luego, para la clase a la cual pertenece, y, finalmente, para la sociedad; incrementos leves de riesgo sólo se justificarían si existiera una fundamentación científica o médica superior ${ }^{30}$.

B. Los beneficios potenciales a los sujetos individuales o a la sociedad se maximizan ${ }^{31}$.

\footnotetext{
Ibíd, Guideline 8.

Ibíd, Guideline 9.

Ibíd, Guideline 8 .
}

En investigación clínica se busca el beneficio de los sujetos de investigación y de pacientes similares.

C. Los beneficios potenciales son proporcionales o exceden a los riesgos asumidos ${ }^{32}$. El beneficio principal es para la sociedad, pero también se considera el beneficio para el individuo.

D. Hay previsión de compensación, indemnización y tratamiento de los sujetos en caso de lesión o muerte atribuibles a los procedimientos de investigación ${ }^{33}$. Los sujetos deben tener garantía de protección por reacciones adversas.

E. Se establecen formas de compensación que se ofrecerán a los investigadores y a los sujetos de investigación por su participación ${ }^{34}$.

F. Hay medidas de protección de sujetos vulnerables, evitando la discriminación ${ }^{35}$.

G. Se establecen criterios para que no se continúe con la investigación, tanto para finalizar el estudio como para que no se prosiga en sujetos individuales ${ }^{36}$.

H. Se proporcionan, en el caso de investigación clínica, los datos toxicológicos y farmacológicos adecuados para el tipo y dosis de medicamento o dispositivo que se pretende implantar, la duración del tratamiento y se garantiza la seguridad de su empleo en el ser humano ${ }^{37}$.

I. En el caso de investigación de medidas terapéuticas o de prevención, se asegura que las intervenciones de la investigación estén

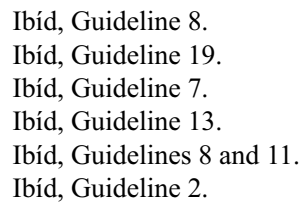


disponibles en el país anfitrión una vez establecida su efectividad y seguridad ${ }^{38}$.

Como regla general, la investigación que involucra a pacientes no debe implicar más que el mínimo riesgo para que sea éticamente aceptable. No obstante, hay excepciones a esta regla cuando ${ }^{39}$ :

1. Hay un beneficio potencial muy grande para un determinado individuo.

2. El sujeto es voluntario sano y entiende bien lo que implica la investigación y desea participar.

3. El riesgo del procedimiento de investigación es mínimo en comparación con los riesgos que ya están comprendidos en el proceso de la misma enfermedad.

4. La enfermedad en estudio es una enfermedad importante.

5. Hay un gran potencial de beneficio en términos del conocimiento adquirible por la investigación.

6. No hay otro medio de obtener tal conocimiento.

\section{Evaluación independiente}

Se deben considerar los siguientes aspec$\operatorname{tos}^{40}$ :

A) Efectuar una evaluación independiente para evitar conflictos de intereses, ya que la responsabilidad es social.

B) Indicar fuentes de financiamiento y administración de recursos y, de este modo, clarificar posibles conflictos de intereses.

Ibíd, Guideline 10.

Ibíd, Guideline 8.

Ibíd, Guideline 2.
C) Los comités de evaluación no pueden aceptar pagos, exclusivamente, para la aprobación o autorización de un protocolo de investigación.

D) Garantizar que el estudio se encuentra dentro de los lineamientos legales.

\section{Consentimiento informado}

El consentimiento informado se justifica por el principio de respeto a las personas y a sus decisiones autónomas. Los individuos sólo participarán cuando la investigación sea compatible con sus valores, intereses y preferencias, ya que la decisión debe ser libre. Es necesario informar sobre la finalidad, riesgos, beneficios y alternativas a la investigación, para que el sujeto tenga los suficientes elementos de juicio para tomar su decisión. Los comités tienen la responsabilidad de evaluar la información escrita que se entregará a los posibles sujetos de investigación o, en su defecto, a su representante legal-, sobre las características del ensayo y la forma en que se proporcionará dicha información, considerando los posibles intereses del paciente.

Los patrocinadores e investigadores tienen el deber $\mathrm{de}^{41}$ :

- Describir el procedimiento de obtención de consentimiento informado teniendo en cuenta factores culturales y de vulnerabilidad, y evaluando la claridad y comprensibilidad para los sujetos.

- Comunicar al posible participante toda la información necesaria para que otorgue un consentimiento debidamente informado.

- Ofrecer al posible participante una oportunidad plena para plantear preguntas y estimularlo a que lo haga.

$41 \quad$ Ibíd, Guidelines 4 and 6 . 
- Excluir la posibilidad de engaño injustificado, influencia indebida o intimidación

- Procurar el consentimiento sólo después de que el posible participante tenga un conocimiento suficiente de los hechos pertinentes y de las consecuencias de su participación, y haya tenido suficiente oportunidad de considerarla.

- Como regla general, obtener de cada posible participante un formulario firmado como prueba de su consentimiento informado.

- Renovar el consentimiento informado de cada participante si ocurren cambios importantes de las condiciones o procedimientos de la investigación, y en el caso de estudios longitudinales de larga duración.

- Para algunas culturas es necesario consultar a líderes de la comunidad, además de obtener consentimiento informado individual.

Para que el proceso del consentimiento informado sea ético, es indispensable considerar que se tengan en cuenta los elementos que lo constituyen:

A) La información. El sujeto debe contar con información suficiente y adecuada. Con este fin, nada mejor que proporcionarle una hoja informativa como soporte de la descripción oral. A este respecto, se considera como información estándar general proveer los siguientes datos ${ }^{42}$ :

1. Razones para incluir al individuo en la investigación.

2. Propósito y objetivos de la investigación.

3. Características de la enfermedad y su pronóstico, si se trata de investigación clínica.

Ibíd, Guideline 5 .
4. Declaración de que puede abandonar la experiencia en el momento que lo desee, sin sufrir represalias.

5. Naturaleza de los procedimientos de la investigación: si se trata de invasivos o no, las molestias que producen, entre otras características.

6. Tipo de estudio y características del diseño.

7. Duración esperada de la participación del individuo.

8. Si se trata de un riesgo mayor al mínimo, cómo le serán compensados los posibles perjuicios causados.

9. Accesibilidad a hallazgos de la investigación y a datos personales. En algunos casos, el comité de evaluación ética puede aprobar no revelar datos, temporal o permanentemente; en este caso se deben ofrecer razones.

10.Riesgos y beneficios implicados para el individuo y para la sociedad.

11. Disponibilidad de los productos o intervenciones después de finalizado el estudio.

12.Intervenciones o tratamientos alternativos.

13. Medidas para asegurar la confidencialidad y privacidad, y sus límites.

14.Patrocinadores de la investigación, afiliación institucional de los investigadores y naturaleza y fuentes del financiamiento.

15.Posibles usos de registros médicos y muestras biológicas para investigación.

16.Posible uso futuro de muestras biológicas y potencial comercial.

17.Si el investigador es también médico del sujeto.

18. Grado de responsabilidad de proporcionar servicios médicos. 
19. Compensación y tratamiento por daños recibidos como consecuencia de participar en la investigación o, cuando corresponda, que no habrá compensación.

20. Aprobación por un comité de evaluación ética.

B) La comprensión. El investigador debe asegurarse de que el potencial sujeto de investigación haya comprendido adecuadamente la información, procurarle oportunidad de hacer todas las preguntas que sean necesarias y responder a todas sus preocupaciones ${ }^{43}$. El sujeto debe tener tiempo suficiente para decidir reflexivamente. Un elemento importante de la comprensión es la competencia ${ }^{44}$, determinada por las facultades mentales de conciencia (sentimiento interior por el cual una persona reconoce sus propias acciones), conocimiento (noción interior del bien que debemos hacer y el mal que debemos evitar), inteligencia (facultad de conocer y comprender, aptitud para establecer relaciones entre las percepciones sensoriales o para abstraer y asociar conceptos) y raciocinio (facultad de inferir un juicio desconocido a partir de otro u otros conocidos). Hay cuatro grupos de personas:

\section{Competentes.}

2. Incompetentes por limitaciones de conciencia (pacientes en coma o con muerte cerebral).

3. Incompetentes por limitaciones del raciocinio (niños, enfermos graves, dementes o adultos mayores).

4. Incompetentes por limitaciones de la inteligencia y del raciocinio (deficientes mentales).

Ibíd, Guideline 4.

Ibíd, Guideline 4.
Las pautas consideran indispensable conseguir autorización de un representante legalmente calificado o apoderado en el caso de capacidad limitada para dar consentimiento informado adecuado por incompetencia, con pautas específicas para el caso de niños pequeños ${ }^{45}$, o de adultos con trastornos mentales o conductuales severos ${ }^{46}$. En el caso de niños se debe buscar su asentimiento y, en el caso de adultos con trastornos mentales, su consentimiento, si se puede, en los momentos lúcidos.

C) La libertad de elección. La ausencia de presión, influencia indebida, incentivo, intimidación o coerción para el asentimiento (abierta o encubierta) ${ }^{47}$. Los investigadores no deben exagerar los beneficios esperables (resultado del estudio) para el bien individual del sujeto de investigación. Es necesario procurar que el sujeto no sienta que el cuidado más esmerado y seguro de su salud está condicionado a su participación en la investigación. El consentimiento informado debe garantizar al paciente su libertad para retirarse del estudio en el momento en que lo desee, sin menoscabo de sus derechos a atención médica. El paciente no debe decidir presionado por miedo de que la confianza-lealtad del médico hacia él se modifique por el hecho de abstenerse de participar. Tampoco se debe presionar al paciente en la decisión ofreciéndole sumas de dinero. En algunos casos hay excepciones por la naturaleza de la investigación, por ejemplo, se justifica, científicamente, la necesidad de engaño o retención de información en ciertas investigaciones sociales en que se genera información sensitiva o potencialmente conflictiva para el sujeto ${ }^{48}$; esto debe quedar claro para el comité de evaluación ética.

Ibíd, Guideline 14.

Ibíd, Guideline 15.

Ibíd, Guideline 6 .

Ibíd, Guideline 6 . 
Si se omite el consentimiento informado, hay que asegurarse de que las razones expuestas estén suficientemente respaldadas ${ }^{49}$, por ejemplo: que el diseño de la investigación sólo implica riesgos mínimos, y el requisito de consentimiento informado haría impracticable su realización. Posibles excepciones al requisito de consentimiento informado ( $\sin$ intrusión en la intimidad y sin riesgo para el sujeto) $\operatorname{son}^{50}$ :

1. Examen de muestras anónimas.

2. Investigaciones basadas en registros médicos sobre los cuales se preserve el anonimato.

3. Investigaciones hechas en el manejo de emergencias inesperadas o no programadas.

\section{Respeto por los sujetos inscritos}

Se justifica por múltiples principios, incluido el de beneficencia y el de respeto a la autonomía. Esta norma de respeto a los sujetos que participan en la investigación implica que:

A. Se asegure que el sujeto tendrá derecho a retirarse del estudio en cualquier momento sin perjuicio de que pueda seguir con tratamiento médico ${ }^{51}$.

B. Se garantice la protección de la confidencialidad y se justifiquen las posibles excepciones $^{52}$. Se busque, por ejemplo, omitir información que podría identificar a los sujetos.

C. Se evite todo tipo de coerción ${ }^{53}$.

D. Se proporcione información sobre riesgos $\mathrm{y}$ beneficios $^{54}$.

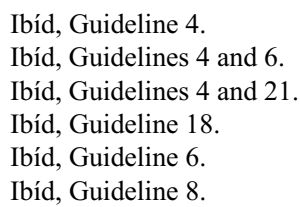

E. Se informe acerca de los resultados ${ }^{55}$.

F. No se descuide el bienestar del sujeto, ya que pueden producirse reacciones adversas ${ }^{56}$.

\section{Problemática de los Comités de Ética en Latinoamerica}

En general, en los países latinoamericanos (aunque existen comités de ética hospitalarios que cumplen funciones de asesoramiento, estudio, docencia y supervisión de la investigación), hay todavía muchas carencias en cuanto a la función de evaluación ética y científica. Debido a que los comités se introducen, en muchos casos, por resolución legal, tienden a ser considerados como una carga burocrática o como una instancia de poder, a veces nombrando como único representante a personas sin formación bioética; tampoco se valora la multidisciplinariedad ni la participación de miembros de la comunidad. Otro problema es la falta de formación de los miembros del comité.

En un estudio efectuado entre 1999 y 2002 por la Unidad de Bioética OPS/OMS, se aplicó una encuesta a todos los Organismos de Ciencias y Tecnología de las Américas (ONCYT) y se llegó a las siguientes conclusiones ${ }^{57}$ :

1. El control ético de los estudios no es entendido o valorado por la mayoría de los investigadores. No está claro tampoco que se intente contribuir al desarrollo social en Latinoamérica.

2. Falta un proceso formal de evaluación ética de propuestas de investigación en algunas instituciones, en las que los comités se reúnen esporádicamente o sólo cuando son, específicamente, requeridos.

\footnotetext{
Ibíd, Guideline 5.

Ibíd, Guideline 21.

Presented at WHO Videoconference DGO/ETH and Regional Focal Points, May $6^{\text {th }}$ (2003).
} 
3. Falta de diferenciación entre los comités de evaluación ética de la investigación y los comités de ética hospitalaria. En muchas ocasiones, los comités de ética hospitalaria que se han ido constituyendo en establecimientos de atención de salud han asumido -sin mucha preparación ni experiencia- la evaluación de protocolos de investigación clínica.

4. Falta de financiación y capacidad administrativa para llevar a cabo las actividades que deben realizar los comités de evaluación ética.

5. La convicción de que los protocolos de investigación externa no necesitan análisis local. En Latinoamérica la mayoría de la investigación es multicéntrica y se lleva a cabo por instituciones del extranjero.

6. No todos los miembros de los comités de evaluación ética conocen, completamente, su función.

7. Falta de educación de los investigadores en ética de la investigación y pautas éticas internacionales para la investigación en seres humanos. Carencia de suficientes profesionales que puedan evaluar ética y científicamente los protocolos. Necesidad de capacitar, urgentemente, a profesionales calificados que conformen Comités de Bioética de la Investigación.

8. Falta de supervisión y mecanismos de control para informar sobre efectos adversos en muchas de las investigaciones que se realizan en Latinoamérica.

9. Hay claras deficiencias respecto de normativas nacionales que regulan la investigación en seres humanos en la mayoría de los países de Latinoamérica. En general, hay Normas Constitucionales que protegen a los ciudadanos, pero solamente como un reconocimiento de diversos derechos que podrían relacionarse, directa o indirectamente, con el tema de la investigación clínica. Las Normas y Códigos Sanitarios referidos, específicamente, a investigación en el ámbito de la salud dan cuenta, principalmente, de procedimientos administrativos para la importación y el registro de fármacos y otros productos biológicos, responsabilidades institucionales y resguardos legales en términos amplios, pero no se refieren a la regulación ética de las investigaciones.

10. Aunque tiende a mejorar, existen pocos Comités Nacionales que supervisen el trabajo de los comités locales y la investigación en general.

\section{Conclusión}

Es necesaria la formación ética en Latinoamérica, tanto de los miembros de comités de evaluación ética y científica como de los investigadores, para que se vaya tomando mayor conciencia de la importancia y relevancia de la ética en la investigación con seres humanos. Por ello, la formación en las pautas éticas internacionales tendría que ser de relevancia en la educación de la comunidad científica.

Debería estimularse la reflexión en el campo de la ética entre los profesionales en Latinoamérica para seguir profundizando en el desarrollo de pautas cada vez más enraizadas en las necesidades y en la cultura propias, al mismo tiempo que se educa a la sociedad en la vivencia de los principios éticos y, así, ayudar a crear mayor conciencia ciudadana sobre la relación entre ciencia y sociedad. Las pautas CIOMS se evalúan cada cierto tiempo, fruto de la reflexión sobre los nuevos desafíos que aparecen en la investigación en países en vías de desarrollo. Sería pertinente que en esta meditación se involucraran cada vez más profesionales y miembros de la sociedad en general, y en particular de Latinoamérica. 